\title{
Stability of cerium oxide on silicon studied by $x$-ray photoelectron spectroscopy
}

\author{
E. J. Preisler, O. J. Marsh, R. A. Beach, and T. C. McGill ${ }^{a}$ \\ T. J. Watson, Sr., Laboratory of Applied Physics, California Institute of Technology, Pasadena, \\ California 91125
}

(Received 9 January 2001; accepted 5 June 2001)

\begin{abstract}
The silicon-cerium oxide interface is studied using $\mathrm{x}$-ray photoelectron spectroscopy. The oxidation and reduction of species at the interface are examined as a function of annealing temperature both in vacuum and oxygen ambient, in order to determine their relative stabilities. By depositing a very thin $\mathrm{CeO}_{2}$ film $(\sim 30 \AA)$, the cerium and silicon core level peaks can be monitored simultaneously. The presence of characteristic chemical shifts of the $\mathrm{Si} 2 p$ peak gives information about any $\mathrm{SiO}_{x}$ layer that may form at the interface. The oxidation state of the cerium can be probed from three different areas of the spectrum. From this information we can infer the oxidation state of both the silicon and the cerium. For the first time a complete picture of the interface is obtained. The implications of these findings on the utility of $\mathrm{CeO}_{2}$ in device applications are discussed. (C) 2001 American Vacuum Society. [DOI: 10.1116/1.1387464]
\end{abstract}

\section{INTRODUCTION}

Cerium oxide has shown potential as a heterostructure material for $\mathrm{Si}^{1}$ as a gate dielectric for a $\mathrm{Si}$ metal-oxide semiconductor field-effect transistor, ${ }^{2}$ as an alternative to ferroelectric materials in nonvolatile capacitor memories, ${ }^{3}$ and as an alternative to the standard $\mathrm{SiO}_{2}$-based silicon on insulator technology. ${ }^{4}$ In all of these applications a critical issue is the nature of the $\mathrm{Si}-\mathrm{CeO}_{2}$ interface. It has been shown that an $\mathrm{SiO}_{x}$ layer (see Fig. 1) can form between the $\mathrm{Si}$ and the $\mathrm{CeO}_{2}$, and further that the $\mathrm{CeO}_{2}$ can sometimes be reduced to $\mathrm{CeO}_{2-x}$ in the presence of $\mathrm{Si}^{5}$ These effects are strongly dependent on temperature, oxygen partial pressure, and other growth conditions. The determination of the parameters governing the state of the top two layers in Fig. 1 is essential to delineating the range of application of this oxide.

Thermodynamics provides some guidance to the kinds of layers that we might find. On the one hand, thermodynamics favors the formation of $\mathrm{CeO}_{2}$ over the formation $\mathrm{SiO}_{2}$. The Gibbs free energy of formation of $\mathrm{CeO}_{2}$ from $\mathrm{Ce}$ metal and $\mathrm{O}_{2}$ gas is $-21.08 \mathrm{eV} / \mathrm{molecule}(-2030 \mathrm{~kJ} / \mathrm{mol})$ at room temperature, whereas for $\mathrm{SiO}_{2}$ from $\mathrm{Si}$ and $\mathrm{O}_{2}$ it is $-17.77 \mathrm{eV} /$ molecule $(-1712 \mathrm{~kJ} / \mathrm{mol}){ }^{6}$ However, it is thermodynamically favored for $\mathrm{Si}$ to reduce $\mathrm{CeO}_{2}$ to its suboxide $\mathrm{Ce}_{2} \mathrm{O}_{3}$, forming $\mathrm{SiO}_{2} \cdot{ }^{7}$ While these thermodynamic arguments may help indicate which reactions will or will not occur in bulk materials, an experimental study of what happens at a real interface is needed. Several studies, most recently Hirschauer et $a l .{ }^{8}$ have shed light on the subject by examining high resolution $\mathrm{x}$-ray photoelectron spectroscopy (XPS) of the $\mathrm{Si}$ core levels and the Ce valence levels. In this study we obtain information from almost every part of the XPS spectrum and produce a detailed picture of the interface.

In this work we confirm that a silicon oxide layer, whose oxidation state depends on annealing conditions, forms underneath the cerium oxide over layer (see Fig. 1). We also

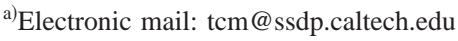

determine that when annealed in vacuum, the cerium oxide will reduce to $\mathrm{Ce}_{2} \mathrm{O}_{3}$ but no further, whereas it will fully oxidize to $\mathrm{CeO}_{2}$ almost immediately when annealed in oxygen.

In Sec. II, we present the details of the preparation of the samples and of the X-ray photoemission spectroscopy experiment. In Sec. III, we present the methods used in our analysis of the data. These techniques include the deconvolution of the core spectra into separate Gaussian peaks, the use of the attenuation of the spectrum by the inelastic collisions of the electrons to determine over layer thicknesses, and an overall analysis of the valence band. Section IV presents our results based on an interpretation of the information given by the core levels treated with the techniques mentioned above. The thermodynamic situation is clarified by comparing data from samples annealed at various temperatures and interpreting them in terms of the oxidation and reduction of the layers at the interface between the silicon and cerium oxide.

\section{EXPERIMENT}

Two samples were prepared by UHV (base pressure 3 $\times 10^{-10}$ Torr) electron beam evaporation of a solid $\mathrm{CeO}_{2}$ source onto polished 3 in. $\mathrm{Si}(111)$ wafers in a Perkin-Elmer silicon molecular beam epitaxy system. Wafers were rinsed in acetone, isopropyl alcohol, and de-ionized water before a final dip in $10 \%$ hydroflouric acid to remove the oxide, and then immediately introduced to vacuum. The growth was performed at low temperature $\left(<400{ }^{\circ} \mathrm{C}\right)$ and without any background oxygen flow in order to minimize oxidation of the substrate prior to growth. The background pressure in the chamber due to oxygen outgassing of the source was close to $1 \times 10^{-5}$ Torr. Thickness was determined by a quartz crystal monitor to be approximately $30 \AA$ for both samples. Samples were also monitored by reflection high energy electron diffraction (RHEED) which showed a transformation from a typical $\mathrm{Si} 1 \times 1$ reconstruction to polycrystalline almost immediately after the source shutter was opened. The samples 


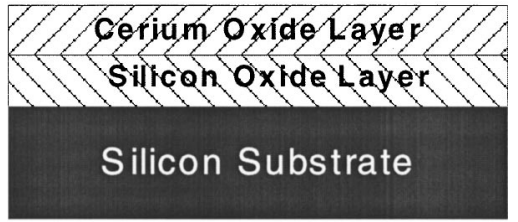

FIG. 1. Schematic of the sample. During deposition we attempt to simply deposit $\mathrm{CeO}_{2}$. However, even during the deposition at roughly $400{ }^{\circ} \mathrm{C}$, one might expect some movement of the atoms and hence the $\mathrm{SiO}_{x}$ layer forms.

were then transferred in UHV to an analysis chamber for performing XPS and back to the growth chamber for subsequent annealing. The analysis chamber is equipped with a monochromated $\mathrm{Al} K \alpha$ x-ray source and a Perkin-Elmer model 10-360 hemispherical analyzer with a position sensitive detector with a minimum resolution of $0.6 \mathrm{eV}$. The samples were radiatively heated for $30 \mathrm{~min}$ at a time at temperatures from 300 to $900^{\circ} \mathrm{C}$. One was always annealed in vacuum and the other in $1 \times 10^{-5}$ Torr of $\mathrm{O}_{2}$ to simulate growth conditions as described above. The temperatures were monitored by optical pyrometer in the range 400$800^{\circ} \mathrm{C}$ which is its range of validity and by thermocouple otherwise. It has been shown that exposure to $\mathrm{Al} K \alpha$ radiation can itself reduce $\mathrm{CeO}_{2},{ }^{9}$ so we performed the $\mathrm{Ce} 3 d$ scans of each data set first. This being said, we noticed no change in the oxidation state before and after exposure except perhaps at the surface.

\section{ANALYSIS TECHNIQUES AND FITTING OF CORE LEVELS}

We have used standard analysis techniques and applied them to the data for each of the core levels ( Si $2 p, \mathrm{Ce} 3 d, \mathrm{O} 1 s$ ) and the valence band edge. In each case we will use the analysis to provide information on the variation in the state of the sample in Fig. 1.

Before examining the individual areas of the XPS spectrum, the methods used to obtain information from the raw data are described here. A Shirley background subtraction ${ }^{10,11}$ was performed on all the raw data. After this, peaks were either integrated to determine their spectral weight or fit with pure Gaussian peaks to deconvolve multiple peaks that are close together in binding energy (BE). The spectral weights of all the peaks were then used to determine either chemical information or the thickness of different layers of material.

To determine a thickness, we use the fact that the signal from electrons emitted from a buried layer is attenuated by inelastic collisions with atoms in the over layer. If the peak from electrons in the buried layer is chemically shifted to a different BE than the peak for electrons in the over layer, the area of the two peaks can be compared. The ratio of the area of the over layer peak to the area of the buried layer peak should thus be related to the thickness of the over layer. More precisely, we use an exponential extinction law where the signal intensity from photoelectrons traveling through various materials decays as
TABLE I. Electron inelastic mean free paths, $\lambda$ in $\AA$. The photoelectron source indicates in which material the detected electrons were created and the over layer material indicates the type of material that the electrons will subsequently have to travel through on their way out the sample. The data for $\mathrm{Si}$ and $\mathrm{SiO}_{2}$ over layers are from Ref. 13, and the data for the cerium species are calculated from the expression given by Tanuma, Powell, and Penn in Ref. 12.

\begin{tabular}{cccc}
\hline \hline \multirow{2}{*}{$\begin{array}{c}\text { Over layer } \\
\text { material }\end{array}$} & \multicolumn{3}{c}{ Photoelectron source } \\
\cline { 2 - 4 } & $\mathrm{Si} 2 p$ & Oxygen $1 s$ & $\mathrm{Ce} 3 d$ \\
\hline Silicon & 30 & $\ldots$ & $\ldots$ \\
$\mathrm{SiO}_{2}$ & 36 & 27 & $\ldots$ \\
$\mathrm{CeO}_{2}$ & 23.8 & 17.9 & 10.9 \\
$\mathrm{Ce}_{2} \mathrm{O}_{3}$ & 27 & 20.3 & 12.3 \\
\hline \hline
\end{tabular}

$$
I=I_{0} \exp \left[-\frac{x}{\lambda}\right],
$$

where $I_{0}$ is related to the cross section for the process that creates the electron, $x$ is the path length the electron has to traverse to leave the sample, and $\lambda$ is the electron inelastic mean free path (IMFP).

As an example, we address the case of the Si $2 p$ peaks. There are two peaks in question, one from the silicon in the substrate and one from the silicon in the $\mathrm{SiO}_{x}$ layer immediately on top of the substrate. The signal from the substrate after traveling through the $\mathrm{SiO}_{x}$ and $\mathrm{CeO}_{x}$ layers is given by

$$
I_{\mathrm{Si}}=\exp \left[\left(\frac{t_{\mathrm{SiO}_{x}}}{\lambda_{\mathrm{SiO}_{x}}}+\frac{t_{\mathrm{CeO}_{x}}}{\lambda_{\mathrm{CeO}_{x}}}\right)\right] \times \int_{0}^{t_{\mathrm{Si}} \rightarrow \infty} I_{0} \exp \left[\frac{-x}{\lambda_{\mathrm{Si}}}\right] d x,
$$

where $\lambda_{\mathrm{Si}}, \lambda_{\mathrm{SiO}_{x}}$, and $\lambda_{\mathrm{CeO}_{x}}$ are the IMFPs of electrons in $\mathrm{Si}$, $\mathrm{SiO}_{x}$, and $\mathrm{CeO}_{x}$, respectively, as described in Table I, and the $t$ 's are the path lengths that the photoelectrons must travel through each material. The strength of the $\mathrm{SiO}_{x}$ signal will be

$$
I_{\mathrm{SiO}_{x}}=\exp \left[-\frac{t_{\mathrm{CeO}_{x}}}{\lambda_{\mathrm{CeO}_{x}}}\right] \times \int_{0}^{t_{\mathrm{SiO}_{x}}} I_{0} \exp \left(\frac{-x}{\lambda_{\mathrm{SiO}_{x}}}\right) d x .
$$

We assume that $\lambda_{\mathrm{SiO}_{x}} \sim \lambda_{\mathrm{SiO}_{2}}$ and, furthermore, that the cross sections for both processes (i.e., $I_{0}$ ) are the same since both peaks come from electrons emitted from $\mathrm{Si}$ atoms. With these assumptions, and taking into account a $45^{\circ}$ detector angle, the final expression for the thickness of the $\mathrm{SiO}_{x}$ layer becomes

$$
t_{\mathrm{SiO}_{x}}=\frac{\lambda_{\mathrm{SiO}_{x}}}{\sqrt{2}} \times \ln \left(1+\frac{\lambda_{\mathrm{Si}}}{\mathbf{r} \times \lambda_{\mathrm{SiO}_{x}}}\right),
$$

where $\mathbf{r}$ is the ratio of $\mathrm{SiO}_{x}$ peak area to substrate peak area. Throughout this work values of $\lambda$ for the cerium species were calculated from the expressions given by Tanuma, Powell, and Penn, ${ }^{12}$ and the values for silicon species were obtained directly from the literature. ${ }^{13}$ All the values used in this study are summarized in Table I. 


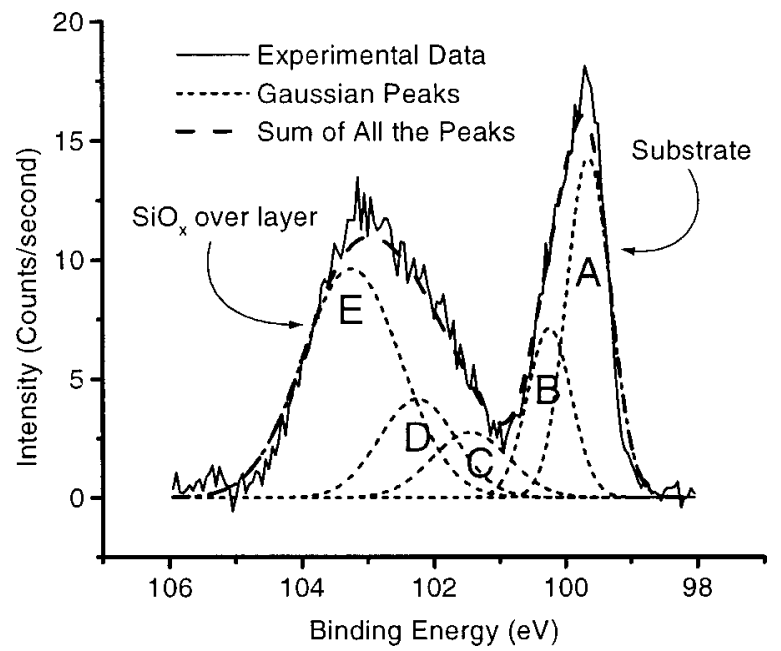

FIG. 2. Example of experimental data (solid line) fitted peaks (dots), and sum of the fitted peaks (dashes) for the Si $2 p$ spectrum. The Si $2 p$ peaks have been deconvolved into five Gaussian peaks $A-E$. The $\mathrm{Si}^{+1}$ peak is too small to be included here. The heavy dashed line is the sum of the contribution of peaks $A-E$ and hence represents an indication of the overall quality of the fit.

\section{A. Silicon $2 p$ core level}

An example of a Si $2 p$ spectrum obtained from the samples described above is shown in Fig. 2 and the peaks used for fitting are summarized in Table II. The Si $2 p$ spectrum was used to gain the following information. First, the position of the Si $2 p$ substrate peak was measured for each data point and compared to data from a bare silicon (111) wafer to be used as a calibration point for the energy scale of all spectra. Second, the large chemical shifts to higher binding energy of $\mathrm{Si}$ atoms in different oxidation states create an obvious satellite peak next to the substrate peak. The relative weights of these two peaks can then be used to monitor the growth of $\mathrm{SiO}_{x}$ species created at the interface. We acknowledge here that what we are calling $\mathrm{SiO}_{x}$ may actually have a finite amount of cerium in it, ${ }^{14,15}$ but it should still be reasonable to assume that the spectrum can be modeled by examining different oxidation states of silicon. Finally, by deconvolving the Si $2 p$ spectrum we can obtain information about in exactly what oxidation state the $\mathrm{Si}$ is.

Good fits $\left(\chi^{2} \leqslant 2\right)$ were found for most spectra by varying only the height and full width at half maximum (FWHM) of these five Gaussian peaks, and letting the positions be determined by the chemical shifts from the literature. ${ }^{16,17}$ Once the fits were performed, the area of oxidized Si species (the

TABLE II. Assignment of the Si $2 p$ Gaussian peaks. $A$ and $B$ are from bare Si. $C-E$ are oxidized states of Si. It is to be understood that each individual oxidized peak represents a double peak similar to the substrate doublet. Chemical shifts are taken from Shallenberger (see Ref. 16).

\begin{tabular}{ccccccc}
\hline \hline Label in Fig. 2 & $A$ & $B$ & $\cdots$ & $C$ & $D$ & $E$ \\
\hline Peak name & $2 p_{3 / 2}$ & $2 p_{1 / 2}$ & $\mathrm{Si}^{1+}$ & $\mathrm{Si}^{2+}$ & $\mathrm{Si}^{3+}$ & $\mathrm{Si}^{4+}$ \\
Relative shift $(\mathrm{eV})$ & 0 & 0.6 & 0.9 & 1.8 & 2.6 & 3.6 \\
\hline \hline
\end{tabular}

TABLE III. Assignments of Ce $3 d$ peaks with $u, v$ notation from Burroughs (see Ref. 21). Shifts represent initial guesses for positions of the peaks with respect to the highest $\mathrm{BE}$ peak for the fitting process.

\begin{tabular}{lcccccc}
\hline \hline \multirow{2}{*}{$d_{5 / 2}$} & & $v_{0}$ & $v$ & $v^{\prime}$ & $v^{\prime \prime}$ & $v^{\prime \prime \prime}$ \\
& Origin & $\mathrm{Ce}(\mathrm{III})$ & $\mathrm{Ce}(\mathrm{IV})$ & $\mathrm{Ce}(\mathrm{III})$ & $\mathrm{Ce}(\mathrm{IV})$ & $\mathrm{Ce}(\mathrm{IV})$ \\
& shift (eV) & -36.1 & -34.1 & -30 & -27.85 & -18.3 \\
& & $u_{0}$ & $u$ & $u^{\prime}$ & $u^{\prime \prime}$ & $u^{\prime \prime \prime}$ \\
$\mathrm{Ce} 3 d_{3 / 2}$ & Origin & $\mathrm{Ce}(\mathrm{III})$ & $\mathrm{Ce}(\mathrm{IV})$ & $\mathrm{Ce}(\mathrm{III})$ & $\mathrm{Ce}(\mathrm{IV})$ & $\mathrm{Ce}(\mathrm{IV})$ \\
& shift (eV) & -17.8 & -15.65 & -13.65 & -9.25 & 0 \\
\hline \hline
\end{tabular}

sum of peaks $C, D$, and $E$ in Fig. 2) was divided by the area of the nonoxidized species (sum of peaks $A$ and $B$ ) to give the ratio $r$ required in Eq. (4).

The relative weights of the four oxidized silicon peaks were then used to determine an average oxidation state for the $\mathrm{SiO}_{x}$ layer. Each of the oxidized peaks may represent a doublet like the substrate peak, but for the level of accuracy we hope to obtain here we assume they are all plain Gaussians. With this assumption, the oxidation state is given by

$$
X_{\text {avg. }}=\frac{2 C+3 D+4 E}{C+D+E} \text {. }
$$

\section{B. Cerium $3 d$ core level}

We observed the change in oxidation state of the cerium by analyzing its $3 d$ spectrum. The cerium $3 d$ spectrum of $\mathrm{CeO}_{2}$ is known for its complexity. Besides the spin-orbit splitting of the $\mathrm{Ce} 3 d_{5 / 2}$ and $\mathrm{Ce} 3 d_{3 / 2}$ there are several other splittings that are caused by a redistribution of the entire

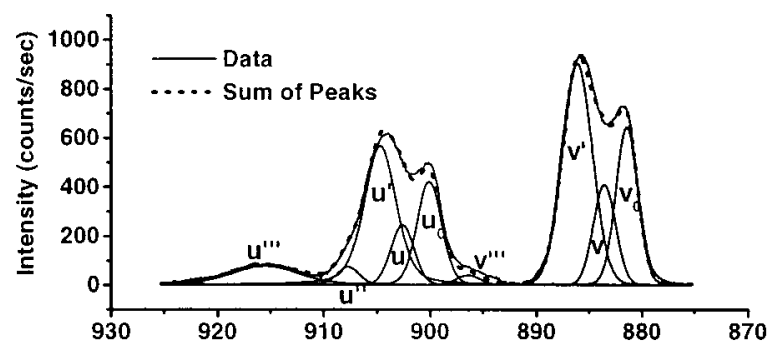

(a)



(b)

FIG. 3. Examples of experimental data of $\mathrm{Ce} 3 d$ spectra and their deconvolution into Gaussian peaks. The labeled peaks are the individual Gaussian peaks used to fit the spectrum and the dashed line is the sum of them which should simulate the experimental data. (a) Reduced spectrum close to $\mathrm{Ce}_{2} \mathrm{O}_{3}$ and (b) a well oxidized spectrum close to $\mathrm{CeO}_{2}$. 
energy spectrum after a core hole is created. This phenomenon is discussed in detail by Fujimori ${ }^{18-20}$ and it is not our intention to examine it further here.

Table III summarizes the ten peaks of the spectrum in the notation of Burroughs et al. ${ }^{21}$ were used for fitting, and Fig. 3 shows two sample spectra with the fitted peaks. Suffice to say, there are four peaks $\left(v_{0}, v^{\prime}, u_{0}, u^{\prime}\right)$ that are derived from $\mathrm{Ce}$ in the (III) oxidation state, and the other six are from the (IV) oxidation state. ${ }^{22}$ Essentially, we are using a version of the method of Romeo et al. ${ }^{23}$ to determine the average oxidation state of the Ce. It has recently been pointed out by Holgado, Alvarez, and Munuera ${ }^{24}$ that a more convenient and accurate way to accomplish this would be via factor analysis, but for the purposes of this study, Romeo's technique is simple and accurate enough. First the whole Ce $3 d$ spectrum is fit to these ten peaks. Hopefully the entire spectrum is well represented by appropriate weighting of the ten peaks. This time we fit the heights, FWHMs, and positions of the peaks. Once we have the fit, we take the individual spectral weights and determine the total weight of $\mathrm{Ce}(\mathrm{III})$ :

$$
\mathrm{Ce}(\mathrm{III})=v_{0}+v^{\prime}+u_{0}+u^{\prime}
$$

and Ce(IV):

$$
\operatorname{Ce}(\mathrm{IV})=v+v^{\prime \prime}+v^{\prime \prime \prime}+u+u^{\prime \prime}+u^{\prime \prime \prime}
$$

and then determine the total fraction of the cerium in the $\mathrm{CeO}_{x}$ layer that is in the (III) oxidation state with

$$
\% \mathrm{Ce}(\mathrm{III}) \text { from } \mathrm{Ce} 3 d=\frac{\mathrm{Ce}(\mathrm{III})}{\mathrm{Ce}(\mathrm{IV})+\mathrm{Ce}(\mathrm{III})} .
$$

\section{Oxygen $1 \mathrm{~s}$ peaks}

The oxygen peaks were used both as another source of information about the cerium oxidation state and as a source of information for the thickness of the cerium oxide. Compared to the cerium spectrum there has been considerably less study devoted to the oxygen spectrum in $\mathrm{CeO}_{2} \cdot{ }^{25} \mathrm{We}$ do know that the $1 s$ peak from oxygen atoms in a $\mathrm{SiO}_{2}$ matrix should be at higher $\mathrm{BE}$ than metal oxides such as $\mathrm{CeO}_{2}$ because Si has a higher electronegativity (1.9 on the Pauling scale) than $\mathrm{Ce}$ (1.12). Further, one would expect that $1 \mathrm{~s}$ electrons in oxygen attached to cerium atoms in the (III) oxidation state would be more tightly bound than for cerium in the (IV) state. Thus we are able to deconvolute the oxygen $1 s$ spectrum into contributions from $\mathrm{Si}-\mathrm{O}, \mathrm{Ce}(\mathrm{III})-\mathrm{O}$, and $\mathrm{Ce}(\mathrm{IV})-\mathrm{O}$ bonded species. These are described in Table IV.

Figure 4 shows that indeed the spectrum can be well fit by three Gaussian peaks. The height, FWHM, and position were all varied to obtain the fit. Peak $C$ is attributed to $\mathrm{SiO}_{x}$ species, not surface absorbed oxygen because it is slightly more shifted in BE than the peak attributed to chemisorbed hydroxide species identified by Sundaram, Wahid, and Melendeg ${ }^{25}$ and because it grows so substantially during oxygen annealing. Peak $A$ is from $\mathrm{Ce}(\mathrm{IV})$ species, and peak
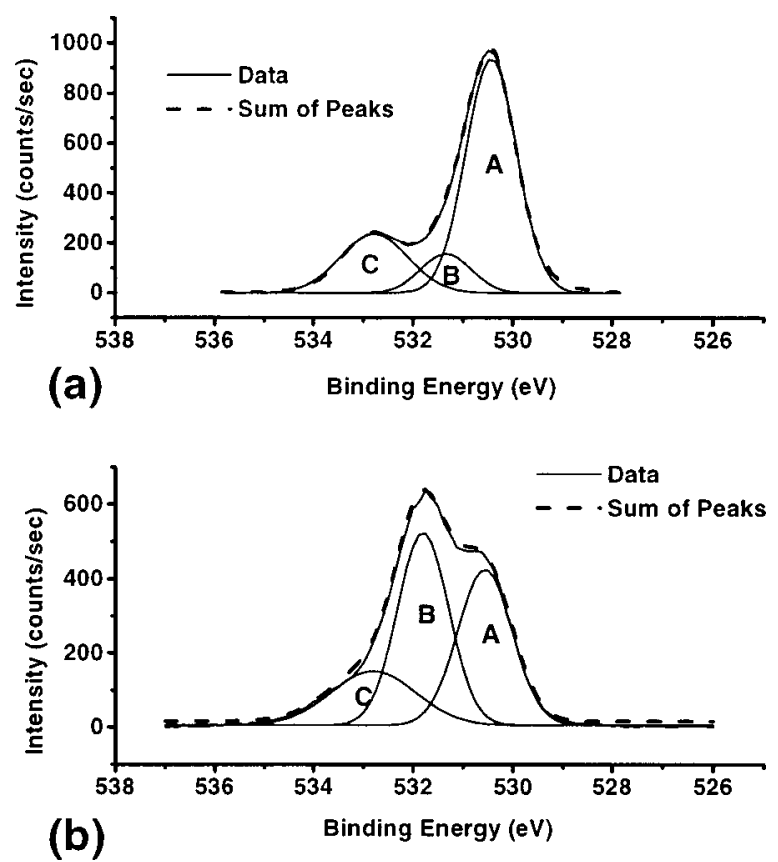

FIG. 4. Examples of experimental data of $\mathrm{O} 1 s$ spectra and their deconvolution into Gaussian peaks. The labeled peaks are the individual Gaussian peaks used to fit the spectrum and the dashed line is the sum of them which should simulate the experimental data. Peak " $A$ " is from oxygen bonded to $\mathrm{Ce}(\mathrm{IV})$ atoms, peak " $B$ "' is from oxygen bonded to Ce(III) atoms, and peak " $C$ " is from oxygen bonded to Si. (a) Well oxidized spectrum (after annealing at $700{ }^{\circ} \mathrm{C}$ in $\mathrm{O}_{2}$ ). (b) Reduced spectrum after annealing at $620^{\circ} \mathrm{C}$ in vacuum.

$B$ is from $\mathrm{Ce}(\mathrm{III})$ species. We found that, in general, the peak due to $\mathrm{Ce}(\mathrm{III})$ was a little over $1 \mathrm{eV}$ higher in binding energy that the Ce(IV) peak.

Examining the relative areas of peaks $A$ and $B$ gives another indication of the fraction of the $\mathrm{CeO}_{x}$ that is in the $\mathrm{Ce}(\mathrm{III})$ state

$\% \mathrm{Ce}(\mathrm{III})$ as determined from $\mathrm{O} 1 s$ spectra $=\frac{B}{A+B}$.

Meanwhile, an equation similar to Eq. (4) can be used to determine the total $\mathrm{CeO}_{x}$ over layer thickness

$$
t_{\mathrm{CeO}_{x}}=\frac{\lambda_{\mathrm{CeO}_{x}}}{\sqrt{2}} \ln \left[1+\frac{\lambda_{\mathrm{SiO}_{x}}\left[1-\exp \left(-\frac{\sqrt{2} t_{\mathrm{SiO}_{x}}}{\lambda_{\mathrm{SiO}_{x}}}\right)\right]}{\mathbf{r} \times \lambda_{\mathrm{CeO}_{x}}}\right] \text {, }
$$

where this time $\mathbf{r}$ is the ratio of the area of the silicon-related peak, $A$, divided by the sum of the areas of the Ce-related peaks, $B$ and $C$.

TABLE IV. Oxygen $1 s$ peak labels in Fig. 4 for oxygen in silicon and cerium oxides.

\begin{tabular}{cccc}
\hline \hline Peak name & $\mathrm{O}(A)$ & $\mathrm{O}(B)$ & $\mathrm{O}(C)$ \\
Species bonded to oxygen & $\mathrm{Ce}(\mathrm{IV})$ & $\mathrm{Ce}(\mathrm{III})$ & $\mathrm{Si}$ \\
\hline \hline
\end{tabular}






FIG. 5. Valence band spectra for the sample annealed in vacuum at all temperatures. One fully oxidized $\mathrm{CeO}_{2}$ valence band is shown on the bottom for comparison. The two top spectra show the valence band after the desorption of oxygen and cerium that occurs for anneals in vacuum past $850^{\circ} \mathrm{C}$. All anneals were for $30 \mathrm{~min}$.

\section{Valence band}

We use the valence band as a third method of monitoring the oxidation state of the cerium. As $\mathrm{CeO}_{2}$ is reduced to $\mathrm{Ce}_{2} \mathrm{O}_{3}$, one of the most obvious feature changes in the entire XPS spectrum is the appearance of the Ce $4 f$ level at the edge of the valence band. ${ }^{26-29}$ Because of the extremely low cross section for creating valence band photoelectrons, our $\mathrm{x}$-ray source was not intense enough to enable the resolution of fine features, but we could easily observe the change in shape due to the mixing of this $4 f$ level. While it is unclear that there is any direct linear correlation between the strength of this feature and the oxidation state of the cerium, we present the valence band spectra of the same samples discussed above as a reality check.

Figure 5 shows normalized versions of all the valence band spectra taken for the sample annealed in vacuum. Only one of the spectra from the sample annealed in $\mathrm{O}_{2}$ is shown because there was hardly any change throughout the annealing. It is shown with the others as a reference point for what the valence band should look like for almost fully oxidized $\mathrm{CeO}_{2}$. Obvious features appear on either side of a steady central peak, which we assume is due to $\mathrm{O} 2 p$ valence electrons. These features can be attributed to an increase in mixing of the Ce $4 f$ state (which is in the middle of the band gap for $\mathrm{CeO}_{2}{ }^{26}$ ) with the valence band and perhaps some to $\mathrm{Si}-\mathrm{O}$ bonding.

\section{RESULTS}

The results are shown in Figs. 6-10. We have interpreted our spectrum in terms of the oxide thickness and oxidation state of the Si and Ce after various anneals. All anneals were for a fixed time of $30 \mathrm{~min}$. Finally, we present a detailed model of the interface.

Equation (4) was used to determine the thickness of the $\mathrm{SiO}_{x}$ layer that forms between the cerium oxide and the substrate (see Fig. 1). A summary of the growth of this oxide is shown in Fig. 6 and the corresponding variation of the oxi-

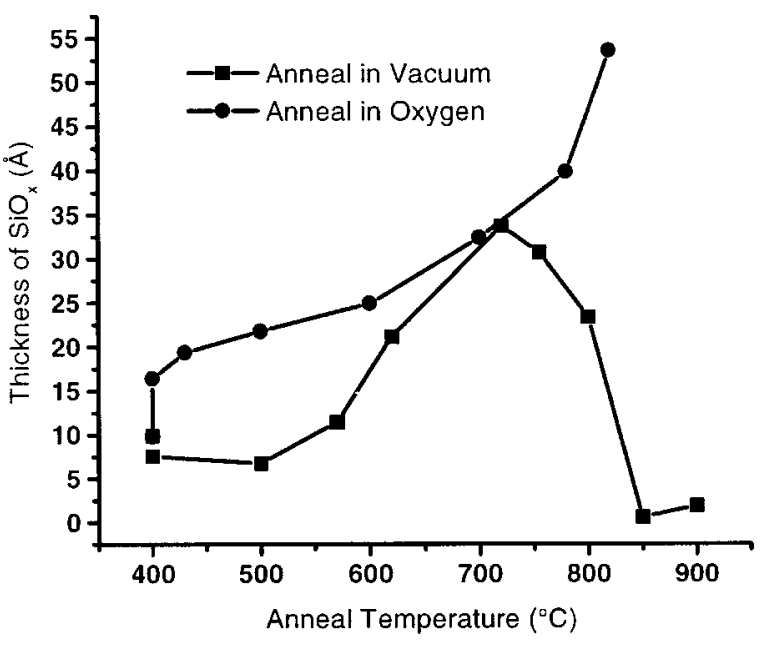

FIG. 6. Measured thickness of the $\mathrm{SiO}_{x}$ layer vs anneal temperature as determined from Eq. (4). The anneals were carried out both in vacuum (squares) and in $\mathrm{O}_{2}$ (circles). All anneals were for $30 \mathrm{~min}$.

dation state is shown in Fig. 7.

The trends for anneals done in oxygen are fairly clear. The $\mathrm{SiO}_{x}$ grows steadily underneath the $\mathrm{CeO}_{x}$ and the oxidation state moves towards (IV), indicating fully oxidized $\mathrm{SiO}_{2}$. It should be noted that the rate of oxidation is actually comparable to that reported for oxidation of bare Si in dry $\mathrm{O}_{2} \cdot{ }^{30}$ In other words, the presence of $\mathrm{CeO}_{x}$ on the surface does not seem to increase the uptake of oxygen by the substrate nearly as much as for pure Ce metal as noted by Hillebrecht et al. ${ }^{14}$

The trends for the anneals done in vacuum, however, are very complicated. Three temperature regimes are apparent.

First, the thickness of the $\mathrm{SiO}_{x}$ stays constant or decreases slightly up to about $500^{\circ} \mathrm{C}$. It will be shown later that this corresponds to a range in which the $\mathrm{CeO}_{x}$ over layer is being reduced. The oxidation state also decreases in this regime as shown in Fig. 7. It would thus seem that at this stage both the

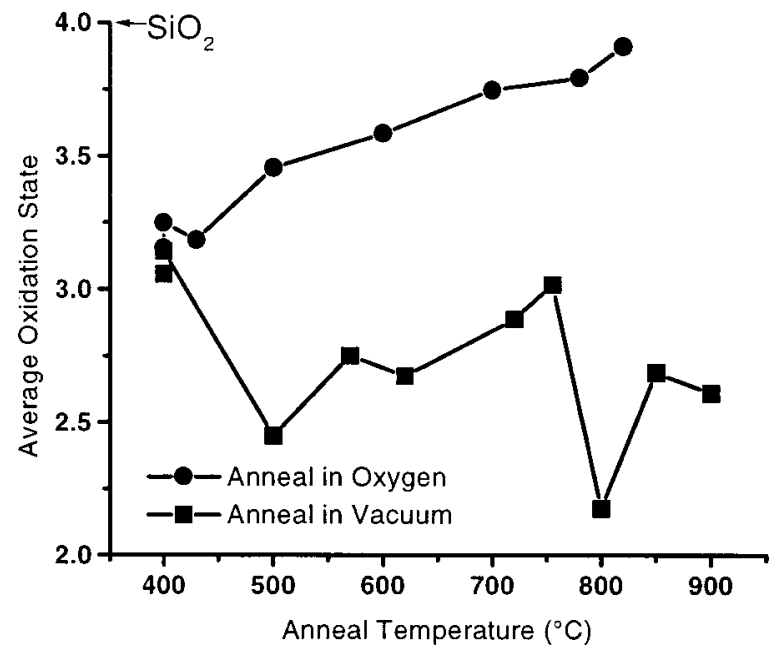

FIG. 7. Measured average oxidation state of the $\mathrm{SiO}_{x}$ vs anneal temperature as calculated from Eq. (5). The anneals were carried out both in vacuum (squares) and in $\mathrm{O}_{2}$ (circles). All anneals were carried out for $30 \mathrm{~min}$. 




FIG. 8. Cerium oxidation state vs anneal temperature as calculated from the $\mathrm{Ce} 3 d$ spectrum. The ordinate is $100 \times \mathrm{Ce}(\mathrm{III}) / \mathrm{Ce}(\mathrm{III})+\mathrm{Ce}(\mathrm{IV})$ on the left and $1.5 \mathrm{Ce}(\mathrm{III})+2.0 \mathrm{Ce}(\mathrm{IV}) / \mathrm{Ce}(\mathrm{III})+\mathrm{Ce}(\mathrm{IV})$ on the right, with quantities defined in Eqs. (6) and (7). The squares are data from sample annealed in vacuum. The circles are for sample annealed in $\mathrm{O}_{2}$. All anneals were carried out for $30 \mathrm{~min}$

silicon and cerium oxides are losing oxygen. A possible explanation for this is that neither the Ce nor the $\mathrm{Si}$ is in a fully oxidized or stable state. The $\mathrm{Si}$ is on average in the (III) oxidation state (see Fig. 7) and Ce is somewhere in between (III) and (IV) (see Fig. 8). These are obviously less thermodynamically stable states than the full oxides, which might mean that some of the oxygen is not bound well enough to prevent outgassing.

Second, from 500 to $720{ }^{\circ} \mathrm{C}$, the $\mathrm{SiO}_{x}$ grows rapidly. In this stage the $\mathrm{Si}$ is clearly reducing the cerium because the $\mathrm{SiO}_{x}$ is growing at the expense of the oxidation state of the $\mathrm{Ce}$ (see Fig. 8). Except for perhaps an anomalous data point at $620^{\circ} \mathrm{C}$, the oxidation state of the $\mathrm{Si}$ increases as the layer grows but never reaches the full (IV) oxidation state of $\mathrm{SiO}_{2}$.

Third, at $720^{\circ} \mathrm{C}$ and above, the $\mathrm{SiO}_{x}$ begins to recede until it totally disappears at $850^{\circ} \mathrm{C}$. Both the oxygen and most of the cerium in the $\mathrm{CeO}_{x}$ over layer also leave the surface in this range. This behavior has been reported earlier by Hirschauer et al., ${ }^{8,31}$ but it is unclear whether this is the same decomposition that Yamamoto et al. ${ }^{32}$ mention as occurring at $690^{\circ} \mathrm{C}$. We also noted two RHEED reconstructions during this regime. At $850{ }^{\circ} \mathrm{C}$ the surface looks very much like a $\operatorname{Si}(111) 1 \times 1$ surface which could be the substrate with the unoxidized $\mathrm{Ce}$ that is left simply conforming to the underlying lattice. At $900{ }^{\circ} \mathrm{C}$ at least two distinct periodicities appear in the RHEED pattern which might be the Ce $2 \times 2 / \sqrt{3} \times \sqrt{3}$ reconstruction mentioned by Hirschauer. More study clearly needs to be done on this phenomenon to quantify it. It should be pointed out here that we were able to continue e-beam $\mathrm{CeO}_{2}$ growth on the interface at this point with good success, obtaining a strong $\mathrm{CeO}_{2} 1 \times 1$ pattern. So whatever species are left at the interface after this transition may actually facilitate further growth.

The oxidation state of the $\mathrm{Ce}$ is also important in giving a clear picture of the state of the layers. First we use the data from the $\mathrm{Ce} 3 d$ peaks to determine the oxidation state. Figure 8 shows the percentage of material that is in the $\mathrm{Ce}$ (III) state, as calculated from Eq. (8), as a function of anneal
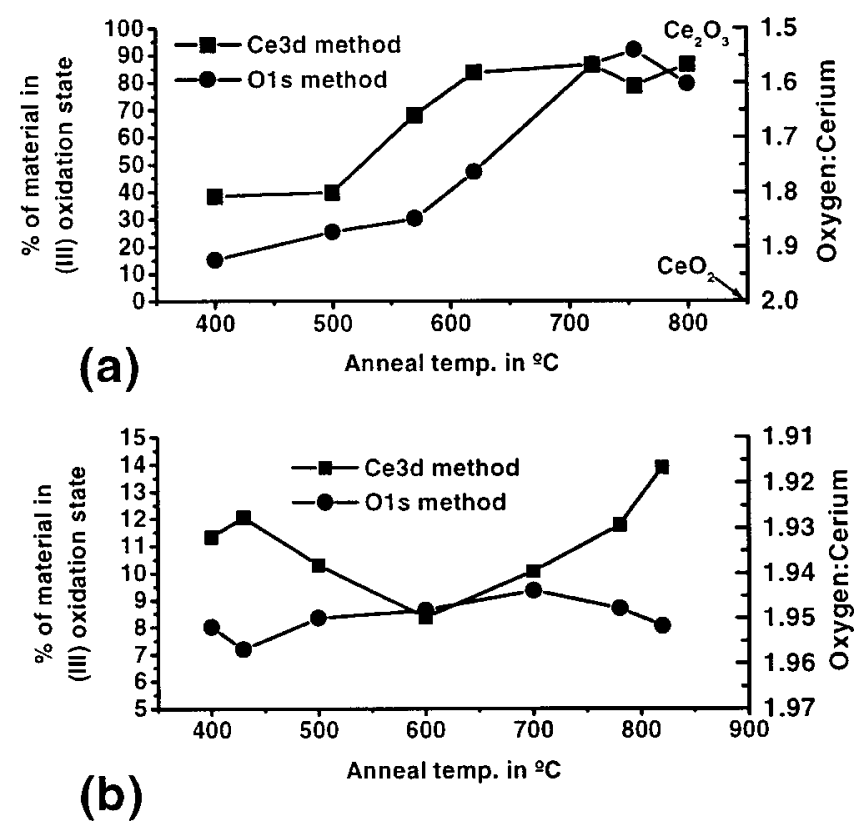

FIG. 9. Oxidation state as determined from the $\mathrm{O} 1 s$ core levels (circles) vs anneal temperature. The ordinate is $100 \times \mathrm{Ce}(\mathrm{III}) / \mathrm{Ce}(\mathrm{III})+\mathrm{Ce}(\mathrm{IV})$ on the left and $1.5 \mathrm{Ce}(\mathrm{III})+2.0 \mathrm{Ce}(\mathrm{IV}) / \mathrm{Ce}(\mathrm{III})+\mathrm{Ce}(\mathrm{IV})$ on the right. The data from Fig. 8 are also plotted for comparison (squares). (a) Sample annealed in vacuum, (b) sample annealed in $\mathrm{O}_{2}$. Please note the very different scale used on the ordinate in this plot (b) vs that in plot (a). All anneals were for $30 \mathrm{~min}$.

temperature in both vacuum and $\mathrm{O}_{2}$. If we naively assume that all $\mathrm{Ce}$ (III) comes from material with a stoichiometry of $\mathrm{Ce}_{2} \mathrm{O}_{3}$, and that all $\mathrm{Ce}(\mathrm{IV})$ comes from material that is $\mathrm{CeO}_{2}$, we can produce a chart of $\mathrm{CeO}_{x}$ stoichiometry versus anneal temperature, which is the alternate ordinate in Fig. 8.

Again the trend in $\mathrm{O}_{2}$ ambient is clear. After one anneal at growth temperature $\left(400^{\circ} \mathrm{C}\right)$ the stoichiometry improves dramatically to about $\mathrm{CeO}_{1.93}$. From there it improves only slightly with further anneals, but it should be noted that even with the $\mathrm{SiO}_{x}$ growing underneath, there is no significant reduction of the cerium. The fact that it never reaches full $\mathrm{CeO}_{2}$ shows either that this method of determining oxidation state is slightly inaccurate or that even fully stoichiometric $\mathrm{CeO}_{2}$ contains some finite amount of $\mathrm{Ce}(\mathrm{III})$ character. It should also be noted that the cerium saturates to a (IV) oxidation state faster than the silicon does (cf. Fig. 7) which indicates that underoxidized cerium perhaps has a higher oxygen affinity than underoxidized silicon.

The trend for anneals done in vacuum agrees with the data shown above for the $\mathrm{Si}$ spectra. That is, very little change is noticed until after $500{ }^{\circ} \mathrm{C}$ at which point the cerium reduces rapidly. One $30 \mathrm{~min}$ anneal at $570{ }^{\circ} \mathrm{C}$ reduces the cerium from $60 \% \mathrm{Ce}(\mathrm{IV})$ to $32 \%$. It continues to reduce only until $620^{\circ} \mathrm{C}$ where one would assume that the stoichiometry has reached $\mathrm{Ce}_{2} \mathrm{O}_{3}$. However, as a converse to the case above, the data suggest that even in fully stoichiometric $\mathrm{Ce}_{2} \mathrm{O}_{3}$ there is some $\mathrm{Ce}(\mathrm{IV})$ character.

The oxidation state of the cerium can also be calculated from the $\mathrm{O} 1 s$ peaks using Eq. (9). Figure 9 again shows the percentage of $\mathrm{CeO}_{x}$ that is in the (III) oxidation state as a 
function of anneal temperature in vacuum and $\mathrm{O}_{2}$. But this time we show the data derived from the $\mathrm{Ce} 3 d$ spectrum along with the data derived from the $\mathrm{O} 1 \mathrm{~s}$ spectrum. The upper panel labeled (a) is for the anneal in vacuum and the lower labeled (b) is for the anneal in $\mathrm{O}_{2}$.

For the sample annealed in vacuum [Fig. 9(a)], the two methods follow the same trend but shifted on the anneal temperature axis. An explanation for this is that the method of using the Ce $3 d$ peaks is far more surface sensitive than the method using the $\mathrm{O} 1 s$ peaks. This is because the IMFP of electrons emitted from Ce $3 d$ levels is far smaller than for those emitted from $\mathrm{O} 1 s$ levels. Thus we have both a surface sensitive and bulk sensitive probe for the oxidation state. This data would suggest that the cerium reduces from the top down rather than first at the interface then proceeding upwards towards the surface because the $\mathrm{Ce} 3 d$ data show the reduction before the $\mathrm{O} 1 s$ data. Further, one can see that both methods show that the amount of cerium reduction saturates. This is most likely the point at which the material is completely reduced to $\mathrm{Ce}_{2} \mathrm{O}_{3}$ since there is no evidence for any stable cerium oxides with less oxygen. ${ }^{33}$ The strange desorption of oxygen and cerium that occurs past $800{ }^{\circ} \mathrm{C}$ obscures any further reduction action that we might have been able to observe.

For the sample annealed in $\mathrm{O}_{2}$ [Fig. 9(b)], it should first be pointed out that the scale is much smaller, i.e., that all the changes in oxidation state are on a smaller scale by almost an order of magnitude than the changes for the sample annealed in vacuum, so these curves are much more susceptible to error introduced by uncertainty in the fitting process. Nevertheless, it is striking that the two trends look to be almost completely opposite. This would seem to indicate that at first the surface is oxidizing while the region near the interface is being reduced slightly by the silicon. However, past $600^{\circ} \mathrm{C}$ the trend reverses and the surface begins to reduce slightly, possibly in the same fashion as the sample annealed in vacuum. Still there are several strange fluctuations along the two paths, so it is difficult to know what is going on in this sample with the same confidence as with the other sample.

Figure 10 shows real-space pictures of what happens at the interface for both samples during annealing. The total thickness of the $\mathrm{CeO}_{x}$ over layer can be computed from the $\mathrm{O} 1 s$ peaks using Eq. (10), and the thickness of the $\mathrm{SiO}_{x}$ has already been computed from Eq. (4). It is assumed that $45 \%$ of the $\mathrm{SiO}_{x}$ growth is down into the substrate, as in the Deal-Grove model. We use the information about both thicknesses combined with the information about oxidation states to create a picture of all the action happening at the interface during the anneals.

We see that in $\mathrm{O}_{2}$ [Fig. 10(b)] the $\mathrm{SiO}_{x}$ grows steadily and pushes up the over layer, whose thickness remains roughly constant. The $\mathrm{CeO}_{2}$ is fixed in thickness due to the limited amount of $\mathrm{Ce}$. On the other hand, the $\mathrm{SiO}_{2}$ layer continues to grow using up the $\mathrm{Si}$ from the substrate.

In vacuum [Fig. 10(a)], there is a maximum in the thicknesses of both oxides before they go through the desorption transition mentioned above, and the interface "regenerates"



(a)

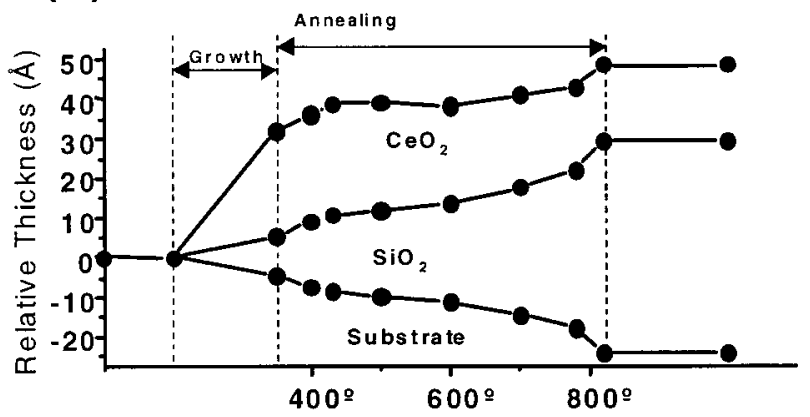

(b)

FIG. 10. Model of the interface obtained from analyzing all of the data. The relative thickness is computed by taking zero to be the surface of the original substrate. The abscissa can be thought of as time. First, the bare substrate is shown closest to the ordinate, then the growth takes place, then successive anneals are performed with the anneal temperature indicated on the abscissa, and finally the far right-hand side of the abscissa is the final state after all annealing. (a) The sample annealed in vacuum, (b) the sample annealed in $\mathrm{O}_{2}$. All anneals were carried out for $30 \mathrm{~min}$.

itself. The large thickness increase of the $\mathrm{CeO}_{x}$ is probably exaggerated by the method used here, but since the density of $\mathrm{Ce}_{2} \mathrm{O}_{3}$ is slightly less than that of $\mathrm{CeO}_{2}$, some thickness increase upon reduction is to be expected. Also, it has been shown recently that under-oxidized cerium oxide nanoparticles undergo a drastic lattice expansion ${ }^{34}$ which may explain the phenomenon we are seeing here. At the highest temperatures all of the oxygen leaves the structure as discussed above, leaving the original Si substrate and a layer of Ce metal.

Finally, we obtain qualitative information about the oxidation state of the cerium from the valence band. Figure 5 shows that as the cerium is reduced, the valence band edge takes on more and more $\mathrm{Ce} 4 f$ character as one would expect from the other methods of determining the cerium oxidation state mentioned above. However, it is difficult to compare this method quantitatively with the other two, since we have no real way of relating the spectral weight of the Ce $4 f$ feature with oxidation state. We merely note here that the $4 f$ character of the valence band increases in the region one would expect it to based on observations of other spectra mentioned above, and it saturates to a value where its height is roughly the same as that of the $\mathrm{O} 2 p$ states. This saturation was also noted in the previous two methods and again seems to be the point of complete cerium reduction to $\mathrm{Ce}_{2} \mathrm{O}_{3}$. The valence band past $800^{\circ} \mathrm{C}$ looks completely different because 
of the desorption mentioned above. It resembles the structure that Hirchauer attributes to the combination $2 \times 2 / \sqrt{3}$ $\times \sqrt{3}$ Ce surface reconstruction. ${ }^{8}$

\section{CONCLUSION}

It is clear that complicated reactions occur at the $\mathrm{Si} / \mathrm{CeO}_{x}$ interface. Some such as the reduction of $\mathrm{CeO}_{2}-\mathrm{Ce}_{2} \mathrm{O}_{3}$ in the presence of $\mathrm{Si}$, are predicted by bulk thermodynamic arguments. However, several unique transitions occur such as the loss of oxygen from all the layers when annealed in vacuum past $800^{\circ} \mathrm{C}$ and the reduction of the $\mathrm{CeO}_{x}$ from the surface down to the interface that would not have been expected.

The picture we have of the interface is as follows. Extremely low temperatures are required to keep the surface completely free of $\mathrm{SiO}_{x}$ growth, which implies that a low temperature growth technique such as pulsed laser deposition might be the only way to achieve a true epitaxial interface for heterostructure applications. However, the strange high temperature transition mentioned above may be a pathway to further high temperature growth as a sort of "self-cleaning", interface. Moreover, for buffer layer applications where the $\mathrm{CeO}_{2} / \mathrm{Si}$ interface is not nearly as important as the quality of the $\mathrm{CeO}_{2}$ surface, we have shown that a $\mathrm{SiO}_{2}$ layer slowly forms underneath when annealed in oxygen but does not affect the $\mathrm{CeO}_{2}$ stoichiometry. Finally, we have shown that the emergence of the $4 f$ states in the valence band does indeed echo the reduction of the cerium and saturates when the cerium is fully reduced to $\mathrm{Ce}_{2} \mathrm{O}_{3}$. The movement of this $4 f$ level from a localized state in the band gap towards an extended state in the valence band surely affects the electrical behavior of $\mathrm{CeO}_{2}$ and thus is important to understand for capacitor and nonvolatile memory applications.

\section{ACKNOWLEDGMENTS}

The authors wish to acknowledge helpful technical discussions with R. Grant about the intricacies of XPS and with J. O. McCaldin about the thermodynamics. The authors acknowledge the patient support of the Defense Advanced Research Projects Agency as monitored by the Air Force Office of Scientific Research under Gerald Witt under Contact No. F49620-96-1-0021.

${ }^{1}$ J. Jones, E. Croke, C. Garland, O. Marsh, and T. McGill, J. Vac. Sci. Technol. B 16, 2686 (1998).
${ }^{2}$ A. Frangoul, K. Sundaram, and P. Wahid, J. Vac. Sci. Technol. B 9, 181 (1991).

${ }^{3}$ L. Kim, J. Kim, D. Jung, and Y. Roh, Appl. Phys. Lett. 76, 1881 (2000).

${ }^{4}$ T. Inoue, Y. Yamamoto, S. Koyama, S. Suzuki, and Y. Ueda, Appl. Phys. Lett. 56, 1332 (1990).

${ }^{5}$ T. Chikyow, S. Bedair, L. Tye, and N. El Masry, Appl. Phys. Lett. 65, 1030 (1994).

${ }^{6}$ CRC Handbook of Chemistry and Physics, edited by D. R. Lide (CRC, Boca Raton, FL, 1994), Chap. 5.

${ }^{7}$ E. J. Tarsa, K. L. McCormick, and J. S. Speck, Mater. Res. Soc. Symp. Proc. 341, 73 (1994).

${ }^{8}$ B. Hirschauer, M. Gothelid, M. Davila, and U. Karlsson, Surf. Sci. 464, 117 (2000)

${ }^{9}$ E. Paparazzo, G. Ingo, and N. Zacchetti, J. Vac. Sci. Technol. A 9, 1416 (1991).

${ }^{10}$ M. Seah, Surf. Sci. 420, 285 (1999).

${ }^{11}$ M. Seah, I. Gilmore, and S. Spencer, Surf. Sci. 461, 1 (2000).

${ }^{12}$ S. Tanuma, C. Powell, and D. Penn, Surf. Sci. 192, L849 (1987).

${ }^{13}$ S. Tanuma, C. Powell, and D. Penn, Surf. Interface Anal. 11, 577 (1988).

${ }^{14}$ F. Hillebrecht, M. Ronay, D. Rieger, and F. Himpsel, Phys. Rev. B 34, 5377 (1986).

${ }^{15}$ Z. Wu, D. Huang, X. Yang, J. Wang, F. Qin, J. Zhang, and Z. Yang, Vacuum 49, 133 (1998).

${ }^{16}$ J. Shallenberger, J. Vac. Sci. Technol. A 14, 693 (1996).

${ }^{17}$ R. Alfonsetti, L. Lozzi, M. Passacantando, P. Picozzi, and S. Santucci, Appl. Surf. Sci. 70-1, 222 (1993).

${ }^{18}$ A. Fujimori, Phys. Rev. B 28, 4489 (1983).

${ }^{19}$ A. Fujimori, Phys. Rev. B 28, 2281 (1983).

${ }^{20}$ A. Fujimori, Phys. Rev. B 27, 3992 (1983).

${ }^{21}$ P. Burroughs, A. Hammett, A. F. Orchard, and G. Thornton, J. Chem. Soc. Dalton Trans. 1976, 686 (1976).

${ }^{22}$ D. Mullins, S. Overbury, and D. Huntley, Surf. Sci. 409, 307 (1998).

${ }^{23}$ M. Romeo, K. Bak, J. ElFallah, F. Lenormand, and L. Hilaire, Surf. Interface Anal. 20, 508 (1993).

${ }^{24}$ J. Holgado, R. Alvarez, and G. Munuera, Appl. Surf. Sci. 161, 301 (2000).

${ }^{25}$ K. Sundaram, P. Wahid, and O. Melendez, J. Vac. Sci. Technol. A 15, 52 (1997).

${ }^{26}$ E. Wuilloud, B. Delley, W. Schneider, and Y. Baer, Phys. Rev. Lett. 53, 202 (1984)

${ }^{27}$ J. Allen, J. Magn. Magn. Mater. 47-8, 168 (1985).

${ }^{28}$ T. Nakano, A. Kotani, and J. Parlebas, J. Phys. Soc. Jpn. 56, 2201 (1987).

${ }^{29}$ A. Pfau and K. Schierbaum, Surf. Sci. 321, 71 (1994).

${ }^{30}$ H. Massoud, J. Plummer, and E. Irene, J. Electrochem. Soc. 132, 2685 (1985).

${ }^{31}$ B. Hirschauer, M. Gothelid, E. Janin, H. Lu, and U. Karlsson, Appl. Surf. Sci. 148, 164 (1999).

${ }^{32}$ Y. Yamamoto, S. Arai, T. Matsuda, M. Satoh, and T. Inoue, Jpn. J. Appl. Phys., Part 2 36, L133 (1997).

${ }^{33}$ Binary Phase Alloy Diagrams, edited by T. B. Massalski (ASM International, Materials Park, OH, 1990), Vol. 2.

${ }^{34}$ S. Tsunekawa, K. Ishikawa, Z. Li, Y. Kawazoe, and A. Kasuya, Phys. Rev. Lett. 85, 3440 (2000). 